\title{
Les collections de l'ancien IFCL, Quels services pour quels usagers? Un état des lieux pour de nouvelles perspectives
}

\section{Valentina DE MONTE}

\section{(2) OpenEdition \\ Journals}

Édition électronique

URL : http://journals.openedition.org/transtexts/508

DOI : 10.4000/transtexts.508

ISSN : 2105-2549

Éditeur

Gregory B. Lee

\section{Référence électronique}

Valentina DE MONTE, « Les collections de l'ancien IFCL, Quels services pour quels usagers ? Un état des lieux pour de nouvelles perspectives », Transtext(e)s Transcultures 跨文本跨文化 [En ligne],

9 | 2014, mis en ligne le 02 septembre 2015, consulté le 20 avril 2019. URL : http:// journals.openedition.org/transtexts/508; DOI : 10.4000/transtexts.508

Ce document a été généré automatiquement le 20 avril 2019.

() Tous droits réservés 


\title{
Les collections de l'ancien IFCL, Quels services pour quels usagers? Un état des lieux pour de nouvelles perspectives
}

\author{
Valentina DE MONTE
}

1 La constitution d'un Fonds chinois au sein de la Bibliothèque municipale de Lyon est intimement liée au sort du patrimoine écrit de l'IFCL, à savoir sa collection d'imprimés constituant l'ancienne bibliothèque destinée aux 473 étudiants chinois qui vinrent faire leurs études en France entre 1921 et 1946 ainsi que sa collection d'archives.

2 Le déplacement de ces collections depuis 2 endroits différents de stockage (le Fort SaintIrénée, siège de l'Institut, et l'Université Lyon 3), débuté en 1974 et réalisé par transferts successifs s'étalant sur plusieurs années, fut motivé par la nécessité de mettre à l'abri dans un endroit convenable un patrimoine déjà menacé dans son intégrité physique, voire intellectuelle ${ }^{1}$.

3 Depuis, les collections chinoises à la Bibliothèque municipale de Lyon n'ont cessé de s'accroître (par le biais de dons, dépôts, échanges et acquisitions onéreuses) ${ }^{2}$ en se développant dans une bibliothèque publique française au-delà de ce que l'on pouvait raisonnablement espérer et envisager au départ. Le Fonds chinois compte aujourd'hui quelque 60000 documents (monographies et périodiques), a un statut patrimonial et, par conséquent, est rattaché au Fonds ancien, un ou deux bibliothécaires (en fonction des aléas du recrutement des personnels spécialisés) ${ }^{3}$ en assurent aujourd'hui la gestion.

\section{Nature de la collection déposée et son traitement}

4 Ce qui est couramment désigné comme "Fonds de l'Institut franco-chinois de Lyon » au sein du Fonds chinois de la Bibliothèque municipale de Lyon recouvre deux gros ensembles documentaires : 
5 La Bibliothèque de l'IFCL, seul exemple identifié de bibliothèque constitué par et pour des étudiants chinois se trouvant temporairement en pays étranger, est elle-même structurée en une section en langues occidentales et une section en langue chinoise.

6 - La section en langues occidentales compte quelque 15000 volumes de livres et environ 150 titres de périodiques. Cette section n'a pas fait à ce jour l'objet d'un traitement spécifique, et pourtant, comme dans le cas de la section chinoise, elle pourrait fournir des éléments fort intéressants de réflexion quant aux instruments à la disposition des étudiants chinois au Fort St-Irénée (siège de l'Institut) pour leur formation scolaire et universitaire, ainsi qu'intellectuelle.

7 - La section chinoise compte quelque 10000 volumes reliés « à la chinoise » datant de la fin du XIX et du début du XXe siècle; environ 4000 volumes de livres «modernes » ${ }^{4}$, imprimés entre 1900 et $1940 ; 425$ titres de périodiques en chinois d'époque républicaine (1911-1949) ; 73 titres de périodiques en langues occidentales d'avant 1949, ayant trait aux études chinoises et publiés en Europe pour la plupart.

8 Les Archives de l'IFCL - s'étalant sur quelques $25 \mathrm{~m}$. linéaires - comptent des documents manuscrits ou tapuscrits, en français ou en chinois majoritairement, parfois les deux langues ensemble, et portent sur la fondation, la gestion ainsi que la dissolution de l'Institut après quelques 30 ans d'existence. Leur structuration reflète de très près le mode de fonctionnement de l'établissement, qui bénéficia d'une double gestion, assurée par les représentants de l'Association universitaire franco-chinoise (pour la partie française) et par les dirigeants chinois, agissant sur en pied d'égalité en fonction des charges attribuées. Ce Fonds constitue un précieux témoignage sur l'histoire de l'établissement et son fonctionnement, le suivi de la scolarité des étudiants, les relations avec les établissements fréquentés par les jeunes Chinois. Il conserve trace des rapports avec les autorités françaises, des relations avec des institutions et personnalités diverses en Chine et en France, voire en Europe. On y trouve des photographies, des sceaux, des diplômes chinois, des tiroirs de fiches bibliographiques et d'autres petits objets. Au coffre, sont également conservés des imprimés rares et précieux: des numéros de revues (éditées tant par l'Institut que par des Chinois en France), des coupures de presse chinoise et française, ainsi que les thèses soutenues par les étudiants de l'Institut - du moins celles qui ont pu être identifiées, qui sont au nombre de 126 à ce jour et remises soit par les étudiants eux-mêmes à l'époque, soit signalés par leurs descendants à une époque très récente. En effet, l'Institut lui-même n'étant pas un établissement diplômant, tous les étudiants se formèrent dans les établissements d'enseignement français, et $1 / 3$ d'entre eux ont achevé leur cursus d'études en soutenant une thèse devant un jury français.

\section{Traitement des collections de l'Ancien IFCL}

Pour ce qui est de la bibliothèque, la priorité a été donnée au traitement des ressources documentaires imprimées en langue chinoise vs la section en langues occidentales citée plus haut. Leur traitement matériel (tri, cotation et mise aux rayons) des monographies et de la riche collection des périodiques de l'époque républicaine est désormais bien achevé et nous arrivons à la phase finale du traitement intellectuel (catalogage) des imprimés conservés. 
10 Ainsi, un inventaire détaillé de la collection des périodiques est accessible en ligne sur le portail du Fonds chinois ${ }^{5}$, à côté d'un inventaire sommaire des 10000 volumes de livres reliés à la chinoise ${ }^{6}$.

11 Pour la section de livres « modernes », en plus du tout premier catalogue papier publié en 1995 par Jean-Louis Boully ${ }^{7}$, leur référencement en bi-écriture (chinois-caractères latins) est disponible, dès 1997/1998, à l'OPAC (Online Public Access Catalog) de la Bibliothèque municipale de Lyon ${ }^{8}$, à l'instar de tout autre document imprimé du Fonds chinois.

$12 \mathrm{Si}$, à l'heure actuelle, et après un pré-traitement, le catalogage d'un lot d'ouvrages littéraires étrangers traduits en chinois au début du $20^{\mathrm{e}}$ siècle est en cours (286 titres, dont 73 catalogués à ce jour, soit $25 \%$ ), deux derniers lots sont en attente de catalogage : l'un de manuels scolaires du début de l'époque républicaine, l'autre de livres en langue japonaise.

13 La gestion documentaire du fonds d'archives a pu être entamée après avoir réuni le fonds dans sa totalité, par une réorganisation physique aux rayons de tous les dossiers. À ma connaissance, les derniers transferts des dossiers d'archives à la Bibliothèque municipale de Lyon datent des années 1980.

14 L'absence d'un inventaire détaillé à l'entrée des collections à la Bibliothèque n'a pas facilité la tâche pour s'orienter aisément au sein de ce corpus. Néanmoins, dès 1987 une liste précise des 473 étudiants régulièrement inscrits à l'Institut, avec leur date de naissance, province d'origine, date d'entrée et de sortie de l'établissement lyonnais, filière d'études choisie lors de l'inscription, a fait l'objet d'une publication papier, précédée de la liste des thèses de doctorat soutenues devant un jury français9. Ces informations sont également disponibles en ligne sur le site de la Bibliothèque municipale de Lyon $^{10}$.

Les dossiers individuels des étudiants ont été réorganisés aux rayons suivant cette liste reconstituée et leur conditionnement et foliotation se poursuit de manière régulière.

Dans le respect du principe de la «provenance» très cher à l'archivistique, l'étape suivante a porté sur un travail d'identification des dossiers relevant de la gestion française vs. ceux relevant de la gestion chinoise avec une attention particulière quant à l'identification - dans la mesure du possible - de leur producteurs. J'insiste ici sur la nécessité d'assurer une gestion de ces archives en réalisant un plan de classement global qui puisse refléter au plus près le mode de fonctionnement de l'Institut lui-même.

17 Après analyse étendue du fonds, l'engagement en 2009 d'une stagiaire, Mlle Émilie Néel, très patiente et très efficace, s'est avéré fort précieux pour la réalisation d'un inventaire sommaire, avec un bref descriptif des dossiers relevant de la "gestion française» de l'Institut, ainsi qu'à un plan de classement, qui a été réalisé sous Word, pour ce qui est de cette section française des archives.

18 La section relevant de la gestion chinoise est moins avancée, pour des raisons purement 'techniques', mais elle se poursuit de manière régulière.

19 Il est toutefois utile de signaler les entraves au traitement qui ont été identifiées et qui peuvent être ainsi brièvement résumées :

20 sur le plan matériel, en plus des manipulations nécessaires au conditionnement et à la mise en conservation des documents : datation délicate des pièces chinoises (non datées ou de date incertaine notamment pour ce qui est de l'année de production), identification 
incertaine des producteurs de certains dossiers, déchiffrage mal aisé de l'écriture cursive chinoise ;

21 sur le plan intellectuel global : nécessité de réfléchir davantage à la structuration d'un plan de classement propre à la nature de la "gestion chinoise », qui se démarque de la structuration donnée aux documents relevant de la "gestion française». A une organisation que l'on pourrait qualifier de 'thématique' (par sujet) des dossiers propres de la gestion française - et pour laquelle un plan de classement a aisément pu être conçu - fait pendant une organisation que je qualifie de 'personnalisée', voire hybride des dossiers produits par les représentants chinois de l'Institut, où coexistent des dossiers organisés par sujet et des dossiers sans aucune structuration apparente (de producteurs, ou de date, ou de sujet). Une certaine logique a pu néanmoins être discernée, quoique faible et imparfaite, pour que la réalisation d'un plan de classement unique puisse enfin voir le jour rapidement ${ }^{11}$.

22 La «conclusion" - si l'on peut dire... - du travail de traitement matériel et intellectuel par le personnel du Fonds chinois trouvera son aboutissement naturel dans la mise en ligne de l'inventaire des archives de l'Institut sur le site de la Bibliothèque municipale de Lyon, à l'instar des fonds patrimoniaux (d'archives, manuscrits et éphémères) qui y sont conservées et déjà traitées, (en XML, avec la DTD «Description archivistique encodée » (ou, en anglais, Encoded Archives Description (EAD) $)^{12}$. Cette mise en ligne ne pourra que représenter une valeur ajoutée de taille aux services déjà offerts à nos usagers.

\section{Quels services pour quels usagers ?}

Quant à cet aspect, il est utile de souligner en préambule que les collections de l'IFCL sont conservées au sein d'une bibliothèque publique française. C'est précisément ce qui explique le double axe (souvent considéré, à tort, comme l'association de deux extrêmes quelque part inconciliables) des actions menées au bénéfice à la fois du grand public et d'un public d'experts et de scientifiques ${ }^{13}$. Ces actions concernent bien sûr non seulement les collections de l'IFCL, mais plus largement toutes les collections en langue chinoise conservées au sein de notre bibliothèque. Aussi, pour le personnel en charge du Fonds chinois un certain... éclectisme s'avère nécessaire pour parvenir à satisfaire les attentes d'un public généraliste, qui souvent ne connait pas un mot de chinois, et pour lequel entre en jeu d'emblée la capacité à agir en quelque sorte en tant que "médiateur entre cultures ", et les attentes des sinologues et spécialistes, qui, elles, dépassent largement le cadre d'un service de bibliothèque publique française. La politique très active de notre bibliothèque envers le patrimoine - une mission très importante parallèle et complémentaire à celle plus traditionnelle de prêt et de renseignement au grand public a certainement été, et demeure, un atout majeur pour ces collections. Très bénéfique en ce sens, a été le rattachement en 1997 du Fonds chinois au Fonds ancien de la Bibliothèque municipale de Lyon (l'un des plus riches de France, quantitativement et qualitativement), qui a enfin enlevé toute incertitude quant à son statut - très fluctuant pendant de longues années ${ }^{14}$, et par conséquent ses missions.

24 Ces publics ont une origine locale (Lyon, France), mais aussi internationale. Que ce soit dans le cadre du renseignement à distance ou du suivi de près de nos lecteurs sur place, nous nous efforçons d'être à l'écoute des besoins exprimés avec pour objectif de leur fournir un service « sur mesure » en termes d'accueil, d'orientation et assistance. 
Pour ce qui est plus spécifiquement des collections de l'IFCL, voici quelques éléments qui caractérisent nos usagers : ces collections sont consultées majoritairement par des étudiants (lycéens, mais très peu d'étudiants universitaires...!), par des chercheurs (à partir du niveau M1) et par des spécialistes. Pour la plupart, ils ne sont pas basés à Lyon et nombreux sont ceux qui viennent de l'étranger (8 nationalités différentes originaires de 3 continents). Ils s'adonnent à un travail intensif sur plusieurs jours d'affilée; consultent à la fois le fonds d'archives et d'imprimés de l'ancien IFCL; gardent le plus souvent un contact professionnel avec les bibliothécaires à distance et au fil des années. Aussi, à ces usagers, en 2011, ont été communiqués 646 documents imprimés ainsi que 220 unités (dossiers) d'archives (pour un total de 11298 feuillets). En plus des consultations individuelles en salle de lecture, les collections de l'Institut franco-chinois attirent également des délégations chinoises, composées de représentants officiels d'institutions culturelles et/ou de recherche, voire des professionnels des médias chinois (presse, télévision, radio), ou encore des descendants des fondateurs ou des anciens étudiants de l'Institut. Aussi, dans le cadre de ces visites très ciblées et à haute teneur scientifique, nécessitant de recherches pointues en magasin en fonction de besoins le plus souvent très précis, en 2011, ont été communiqués, lors de 7 visites au total, 257 documents imprimés et 2688 pièces d'archives du Fonds de l'IFCL.

Pour terminer avec les chiffres, signalons aussi qu'environ 2250 reproductions ont été demandées par les lecteurs à partir de 1252 documents des collections de l'ancien Institut franco-chinois.

Ces informations issues des données récoltées d'une manière systématique en 2011 confirment des points déjà bien identifiés - quoique d'une manière beaucoup plus empirique - jusque là.

Les nouvelles technologies appliquées à l'information et l'avènement d'Internet ont largement contribué à une meilleure gestion et à une plus large visibilité de nos collections. En plus du traitement informatisé des ressources documentaires, cité cidessus, la création d'un portail « Fonds chinois » sur le site de la Bibliothèque municipale de Lyon a permis la mise en place d'un autre moyen efficace pour accomplir nos missions d'information aux usagers et de valorisation des fonds conservés. Très sensibles à la dimension internationale de notre public, toutes les pages de notre portail «Fonds chinois " sont, dès 2004, publiées en ligne en version trilingue (français, anglais et italien).

29 Cette double dimension locale et internationale a, en parallèle, également investi les nombreuses actions de valorisation menées au fil des années. Au niveau local, je citerai les actions visant un public généraliste, telles que, par exemple, l'organisation de conférences ou des Heures de la Découverte. Cette dernière formule demeure un très bel exemple d'accès aux contenus des collections patrimoniales, difficilement accessibles par ailleurs au grand public, auquel nous présentons un choix de documents issus de nos fonds et portant sur un sujet spécifique. Ainsi, à partir d'octobre 2011, j'ai animé, entre autres, un cycle de 6 séances centré sur l'histoire des anciens étudiants de l'IFCL. Pour le prêt de documents imprimés du Fonds de l'IFCL, je prendrai pour exemple notre collaboration à l'exposition Roar China! organisée au Museum voor Schone Kunsten de Gent (Belgique) du 17 octobre 2009 au 7 février 2012, à l'occasion d'Europalia. Un corpus de documents imprimés d'époque républicaine dûment sélectionnés côtoyaient ceux conservés au National Art Museum of China de Pékin, au Lu Xun Museum, toujours de Pékin, ainsi qu'au Lu Xun Memorial de Shanghai ${ }^{15}$. 
30 En ce qui concerne les expositions organisées par la Bibliothèque municipale de Lyon sur l'histoire de l'IFCL, citons celle sur pièces originales avec textes en français et en chinois, ouverte à la Bibliothèque de Canton en juin 2004 dans le cadre des Années croisées France/Chine ${ }^{16}$. Les contenus scientifiques produits par le personnel en charge du Fonds chinois à l'époque (Monsieur Jean Louis Boully et moi-même), agrémentés d'une riche iconographie, ont par la suite été largement réemployés, en les ciblant davantage en fonction des besoins spécifiques, pour deux autres opérations d'envergure : la réalisation d'un espace d'exposition permanent dans deux locaux du Fort Saint-Irénée de Lyon, siège de l'ancien Institut, et d'une exposition temporaire hébergée dans le Pavillon de la région Rhône-Alpes à l'Exposition universelle de Shanghai à l'été $2010^{17}$.

31 À l'instar des collections d'imprimés du fonds de l'IFCL, les archives sont non seulement accessibles aux lecteurs mais aussi valorisées dans le cadre de nos actions culturelles et cela bien que leur traitement ne soit pas achevé. Ce dernier reste en perspective l'un des objectifs prioritaires, compte tenu des ressources humaines et techniques disponibles au sein du Fonds chinois ${ }^{18}$.

Afin de prioriser le traitement et rationaliser la gestion du fonds, l'identification des besoins des chercheurs en termes de sources primaires demeure essentielle, contribuant en même temps à fournir aux usagers un service 'sur mesure' de qualité. A défaut de la disponibilité d'un inventaire détaillé du fonds d'archives, c'est l'excellente connaissance des contenus par le personnel du Fonds chinois qui a permis à ce jour de fournir aux lecteurs ce dont ils ont besoin pour leurs recherches. Mais l'équation archiviste = spécialiste est-elle pour autant nécessaire?

À l'heure actuelle, une mutualisation des compétences entre les bibliothécaires en charge du fonds et les spécialistes et les chercheurs dans les domaines de l'histoire de la présence chinoise en France tout comme de l'histoire intellectuelle de l'époque républicaine en Chine, ne peut qu'être la bienvenue, car une telle synergie, et la disponibilité d'une littérature scientifique produite par des chercheurs et des scientifiques, ne pourraient que bénéficier grandement au traitement documentaire et à la valorisation de ce fonds. Les échanges eus avec les chercheurs au fil des années sont en ce sens incontestablement probants $^{19}$.

34 Une question de taille demeure toujours d'actualité : est-il envisageable de mettre sur pied une équipe de chercheurs de différents pays (France, Chine continentale, Taiwan...) travaillant spécifiquement sur les fonds de l'IFCL et en parallèle sur les sources primaires et secondaires conservées à ce jour dans les pays respectifs ? En tant que professionnelle dans le domaine de la gestion documentaire, j'accueillerais très favorablement une telle initiative.

Elle aurait par ailleurs l'avantage de fournir une solide contribution aux réflexions à mener sur le plan scientifique pour tout projet de numérisation concernant le fonds de l'Institut (fonds d'archives et imprimés), car cet aspect impacte d'emblée le choix du corpus de documents à numériser, en fonction des objectifs arrêtés d'une part et, d'autre part, en vue de l'éventuelle complémentarité avec les autres fonds (déjà numérisés ou pas encore) qui sont conservés en France et en Extrême-Orient. Cette réflexion devrait constituer la toute première étape d'un projet de numérisation, d'autant plus qu'il n'est pas très réaliste - me semble-t-il - de considérer comme viable l'option de "tout" numériser d'emblée. 


\section{NOTES}

1. Pour plus de détails, voir ma communication présentée au 72e Congrès de l'IFLA (International Federation of Library Associations and Institutions) à Séoul en 2006: «From the 'Library of Chinese students in France' to the 'Chinese collections of the Lyon municipal library' ", à l'adresse suivante: http://archive.ifla.org/IV/ifla72/papers/085-Monte_trans-en.pdf (versions en français et espagnol également disponibles).

Techniquement, il s'agit d'un dépôt à long terme, l'Université de Lyon 3-Jean Moulin en restant propriétaire. Ce dépôt a été formalisé bien des années plus tard, par une convention signée le 6 juillet 1987 (Contrat de dépôt. Ouvrages, périodiques et documents d'archives issus de la dévolution de l'ancienne Association franco-chinoise) entre les représentants de la Ville de Lyon et de l'Université Lyon 3-Jean Moulin. Les termes de ce dépôt ont été réactualisés par une deuxième convention signée entre les deux institutions en 2011.

2. Le dernier don en date, composé de la bibliothèque ainsi que des archives de Mme Li Chensheng 李尘生 (Danielle Li), ancienne Maître de conférences du Département de chinois de 
l'Université Lyon 3-Jean Moulin, a rejoint nos collections en 2010, s'ajoutant aux Fonds Jacques Guillermaz (1911-1998), Michelle Loi (1926-2002) et Michel Soymié (1924-2002) ; parmi les dépôts, signalons les collections d'imprimés d'époque républicaine de l'Institut des Hautes Études Chinoises du Collège de France et la section chinoise de la Collection jésuite des Fontaines (12000 volumes environ), dont la bibliothèque personnelle d'André d'Hormon (1881-1965); des programmes d'échanges de documents sont en cours avec les Bibliothèques de Shanghai et Canton, en Chine continentale, ainsi qu'avec la Bibliothèque nationale de Taiwan. Un programme d'échanges avec la Bibliothèque de la Ville de Taibei vient en revanche enrichir les collections en langue chinoise du Département Jeunesse de notre bibliothèque. Pour plus de détails, v. le portail $\mathrm{du}$ Fonds chinois sur le site de la Bibliothèque municipale de Lyon à l'adresse suivante : http:// www.bm-lyon.fr/trouver/Fonds_chinois/Fonds_chinois.htm.

3. Le premier professionnel travaillant sur ce fonds a été embauché en 1985 seulement à hauteur de 5 h par semaine. Le premier emploi à temps plein date de 1993.

4. Le terme "moderne", par opposition au corpus de volumes à reliure traditionnelle chinoise (xianzhuang 線裝) est ici utilisé moins pour les contenus que pour se référer aux nouvelles techniques d'impression importées en Chine d'Occident au XIXe siècle, et qui ont radicalement changé la forme du livre imprimé (reliure, mise en page, papier, etc.).

5. À l'adresse suivante : http://www.bm-lyon.fr/trouver/Fonds_chinois/ressources/revueschinoises.html pour les collections en chinois et http://www.bm-lyon.fr/trouver/Fonds_chinois/ ressources/Perio-IFCL-langues-occidentales.pdf pour celles en langues occidentales.

6. À l'adresse suivante : http://www.bm-lyon.fr/trouver/Fonds_chinois/ressources/Tableauinventaire.pdf

7. Ouvrages en langue chinoise de l'Institut franco-chinois de Lyon, Lyon, Bibliothèque municipale de Lyon, 1995.

8. À l'adresse suivante : http://catalogue.bm-lyon.fr/

9. Catalogue des thèses de doctorat des étudiants de l'Institut franco-chinois [suivi de la] Liste des noms des étudiants de l'Institut franco-chinois de Lyon, par Jean-Louis Boully et Danielle Li respectivement, Lyon, Association des Amis des bibliothèques de la Ville de Lyon, 1987.

10. La liste des étudiants est disponible en format PDF à cette adresse : http://www.bm-lyon.fr/ trouver/Fonds_chinois/ressources/IFCL-Liste-etudiants.pdf. Pour la liste des thèses, également en format PDF, cf. : http://www.bm-lyon.fr/trouver/Fonds_chinois/ressources/IFCL-Thesesetudiants.pdf

11. A l'heure à laquelle je reprends cette communication (donnée en janvier 2012) en vue de sa publication (printemps 2013), il est bien regrettable - tant au niveau de la gestion documentaire qu'à celui du service aux usagers - de ne pas avoir pu rentabiliser des années d'études et recherches sur cette section chinoise des archives, en réalisant avant mon départ du Fonds chinois de la Bibliothèque municipale de Lyon en juillet 2012, le plan de classement que j'avais pu concevoir.

12. Dès 2003, Pléade est l'outil opérationnel à la Bibliothèque municipale de Lyon pour le traitement de ces types de fonds. Pour un aperçu de ce qui est déjà disponible, v. http:// www.bm-lyon.fr/trouver/pleade/archives.htm

13. À noter que $30 \%$ des lecteurs du Fonds chinois réalisent des recherches à titre personnel, donc hors du cadre d'un objectif d'étude ou recherche scientifique (année 2011).

14. Je rappelle cela pour souligner en même temps les difficultés initiales à faire sortir de l'ombre les collections de l'IFCL- en les traitant, en les rendant accessibles aux usagers et en les valorisant : reçues à la Bibliothèque après qu'elles furent laissées à l'abandon pendant plus de 20 ans, les spécialistes s'y référaient avec le label de ghost collections.

15. Voir le catalogue de l'exposition : Roar China! Lu Xun, Masereel en de grafische avant-garde in China, 1919-1949 / Lu Xun, Masereel et l'avant-garde graphique en Chine, 1919-1949, par Gladys Fabre, Zhang Xidan et Bruno Fornari, Gent, Museum voor Schone Kunsten, 2009. 
16. Disponible en version bilingue (français-chinois) sur notre portail à l'adresse : http:// www.bm-lyon.fr/lyonetlachine/

17. Le projet d'exposition à Shanghai a été piloté par le Département de chinois de l'Université Lyon 3-Jean Moulin.

18. Aussi, l'objectif de traiter 18000 feuillets (soit $1 / 3$ de plus que les consultations effectuées en 2011) a été inscrit parmi les objectifs de l'année 2012.

19. Le seul ouvrage scientifique qui fait encore référence à ce jour, ayant une approche factuelle et historique de l'Institut franco-chinois de Lyon, est le mémoire de maîtrise en 2 volumes de Philippe Yann, L'Institut franco-chinois de Lyon, un exemple réussi de collaboration en éducation?, sous la direction de Mme Christine Cornet, Université Lumière-Lyon 2, juin 1998.

\section{AUTEUR}

\section{VALENTINA DE MONTE}

Valentina De Monte est l'ancienne conservatrice du Fonds chinois de la Bibliothèque municipale de Lyon. 